\title{
Validation of a BMI cut-off point to predict an adverse cardiometabolic profile with adiposity measurements by dual-energy X-ray absorptiometry in Guatemalan children
}

\author{
Olga Redondo ${ }^{1,2}$, Eduardo Villamor ${ }^{3}$, Javiera Valdés ${ }^{4}$, Usama Bilal ${ }^{1,5}$, \\ Benjamín Caballero ${ }^{6}$, Dina Roche ${ }^{7}$, Fernanda Kroker ${ }^{7}$, Manuel Ramírez-Zea ${ }^{7}$ and \\ Manuel Franco ${ }^{1,5, *}$
}

${ }^{1}$ Social and Cardiovascular Epidemiology Research Group, School of Medicine, University of Alcalá, Alcalá de Henares, Madrid 28871, Spain: ${ }^{2}$ Research Support Unit, La Mancha Centro General Hospital, Alcázar de San Juan, 13600 Ciudad Real, Spain: ${ }^{3}$ Department of Epidemiology, School of Public Health, University of Michigan, Ann Arbor, MI, USA: ${ }^{4}$ Department of Public Health, Medical Faculty, Catholic University of the North, Santiago de Chile, Chile: ${ }^{5}$ Department of Epidemiology, Johns Hopkins Bloomberg School of Public Health, Baltimore, MD, USA: ${ }^{6}$ Center for Human Nutrition, Department of International Health, Johns Hopkins Bloomberg School of Public Health, Baltimore, MD, USA: ${ }^{7}$ INCAP Comprehensive Center for the Prevention of Chronic Diseases (CIIPEC), Institute of Nutrition of Central America and Panama (INCAP), Guatemala 1188, Central America

Submitted 10 October 2013: Final revision received 21 March 2014: Accepted 7 May 2014: First published online 17 June 2014

\begin{abstract}
Objective: To identify a body fat percentage (\%BF) threshold related to an adverse cardiometabolic profile and its surrogate BMI cut-off point.

Design: Cross-sectional study.

Setting: Two public schools in poor urban areas on the outskirts of Guatemala City. Subjects: A convenience sample of ninety-three healthy, prepubertal, Ladino children (aged 7-12 years).

Results: Spearman correlations of cardiometabolic parameters were higher with $\% \mathrm{BF}$ than with BMI-for-age $Z$-score. BMI-for-age $Z$-score and \%BF were highly correlated $(r=0.84)$. The $\% \mathrm{BF}$ threshold that maximized sensitivity and specificity for predicting an adverse cardiometabolic profile (elevated homeostasis model assessment-insulin resistance index and/or total cholesterol:HDL-cholesterol ratio) according to receiver operating characteristic curve analysis was $36 \%$. The BMI-for-age $Z$-score cut-off point that maximized the prediction of $\mathrm{BF} \geq 36 \%$ by the same procedure was 1.5 . The area under the curve (AUC) for \%BF and for BMI data showed excellent accuracy to predict an adverse cardiometabolic profile (AUC 0.93 (SD 0.04)) and excess adiposity (AUC 0.95 (SD 0.02)).

Conclusions: Since BMI standards have limitations in screening for adiposity, specific cut-off points based on ethnic-/sex- and age-specific \%BF thresholds are needed to better predict an adverse cardiometabolic profile.
\end{abstract}

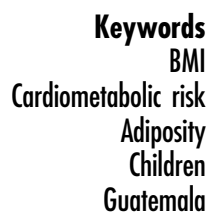

The International Obesity Task Force estimated in 2010 that up to 200 million schoolchildren were overweight; of those, 40-50 million were obese ${ }^{(1)}$. The great increase of childhood overweight/obesity over the last three decades seen in developing countries is now being observed in low- and middle-income countries elsewhere in the world, particularly in urban settings, where racial and ethnic disparities in obesity prevalence exist ${ }^{(2)}$. As an example, Guatemala is experiencing 'the double burden of disease', with the coexistence of chronic malnutrition and an obesity prevalence of $12 \cdot 3 \%$ in pre-adolescent children ${ }^{(3)}$. Half of Guatemalan children aged 8-13 years have at least one component of the metabolic syndrome (MetS). However, it is uncertain whether conventional BMI-based definitions of overweight/obesity appropriately identify children with excess adiposity who are then at cardiometabolic risk ${ }^{(4)}$.

Obesity is a medical condition in which excessive body fat (BF) may impair health and represents the fifth leading risk for death globally ${ }^{(1)}$. Although excess fat is the indicator of obesity, BMI is considered a surrogate measure of $\mathrm{BF}$ to classify individuals as obese ${ }^{(5)}$. Nevertheless, the accuracy of BMI to estimate BF is debatable in childhood because adiposity is affected by growth and stages of maturation $^{(6-8)}$. Moreover, variations in adiposity and its relationship with BMI by sex and race-ethnicity can lead to misclassification of obesity ${ }^{(9)}$. 
The WHO cut-off points to define overweight inadequately estimate percentage body fat $(\% \mathrm{BF})$ in Caucasian populations and different racial groups in Asia ${ }^{(10)}$. Also, it is known that common BMI cut-offs to diagnose overweight have high specificity, but low sensitivity in identifying adiposity, failing to identify half of the people with excess $\% \mathrm{BF}^{(7,11)}$. Despite the significant correlation between \%BF and BMI, children grouped by their BMI do not group similarly based on their $\% \mathrm{BF}^{(8)}$. As BMI does not account for wide variations in BF distribution, it may not correspond to the same degree of adiposity and associated health risks in different individuals and populations ${ }^{(7,12)}$.

Then, it is of relevance to continue studying the relationship between $\mathrm{BMI}$ and \%BF to properly identify the possible components of MetS ${ }^{(13)}$. Several studies have assessed BF cut-offs correlating with biological risk ${ }^{(14,15)}$. Others have evaluated the ability of different BMI cut-off points in childhood to predict cardiovascular risk in midadulthood $^{(16)}$. However, to our knowledge, no study has defined BMI cut-offs based on the metabolic disorders related to adiposity by means of a two-step procedure. Health-care professionals need a more sensitive BMI reference tool for children to enable early detection and to quantify the degree of obesity in the prevention of future cardiovascular health hazards ${ }^{(17-19)}$.

Our objective was to identify a \%BF threshold associated with an adverse cardiometabolic profile and its surrogate BMI cut-off point in a population of Guatemalan children.

\section{Methods}

\section{Participants and setting}

The present study is part of a cross-sectional study carried out by the Comprehensive Center for the Prevention of Chronic Diseases (CIIPEC), at the Institute of Nutrition of Central America and Panama (INCAP). The main objective was to measure the prevalence of cardiovascular risk factors in normal-weight and overweight children in order to understand cardiovascular health in poor Guatemalan children.

Ninety-three healthy, prepubertal, Ladino children (a distinct ethnic group composed mostly of Spanish/ American Indian mestizos), aged between 7 and 12 years, were randomly recruited within two elementary public schools of Mixco in the peri-urban area of Guatemala City (forty-six boys and forty-seven girls). Schools were selected by convenience, for being located in a poor area (children from low- and medium- to low-income families), with the agreement of the principals and teachers. They were similar to the applicant schools that later on would be included in an intervention programme.

Inclusion criteria were: apparently healthy children with BMI within the normal range ( $n 53)$ or overweight $(n 40)$ according to WHO classification (BMI $Z$-score of 0 to 1 for normal weight and $>1$ for overweight) and living in the peri-urban area. Exclusion criteria were: chronic disease, menarche, having a brother/sister already included in the study and undernutrition (WHO BMI $Z$-score $<-2$ ).

INCAP's Institutional Ethics Committee approved the study protocol. Informed written consent was obtained from parents and verbal assent from each child before the tests.

\section{Measurements}

Anthropometry, body composition and cardiometabolic measurements were performed early in the morning on each child by three trained members of the research team at the Physiology and Body Composition Laboratory of CIIPEC, using standard protocols, from August to December 2010. Children, who were accompanied by one parent or tutor, had fasted overnight and had avoided any kind of physical exertion for at least $12 \mathrm{~h}$ before the test. All measures are summarized in Table 1.

\section{Anthropometry}

Weight (kg) was obtained to the nearest $0 \cdot 01 \mathrm{~kg}$ using a calibrated digital scale (Mettler Toledo IND 221; Mettler Toledo Inc., Columbus, OH, USA), with the child wearing a swimsuit. Height $(\mathrm{cm})$ was obtained using a stadiometer (Perspective Enterprises, Portage, MI, USA) calibrated to the nearest $1.0 \mathrm{~mm}$, with the child standing in bare feet with his/her head, shoulders, buttocks and heels leaning against a surface that was at a $90^{\circ}$ angle to the floor. BMI was calculated as weight $/$ height $^{2}\left(\mathrm{~kg} / \mathrm{m}^{2}\right)$. BMI $Z$-scores were evaluated according to the WHO standards ${ }^{(20)}$. Precision was estimated with the mean of two measurements. If the difference between both measurements was $0.5 \mathrm{~kg}$ for weight and $0.5 \mathrm{~cm}$ for height, a third was obtained, and the mean of the two closest was used.

\section{Body composition}

Percentage of trunk fat (\%TF), \%BF and fat mass $(\mathrm{kg})$ were measured by dual-energy X-ray absorptiometry using a Lunar iDXA instrument (GE Healthcare Worldwide, General Electric Company, Bucks, UK) and enCORE 2008 (version 12.30.008) software. This is the most used reference technique to measure fatness owing to its high accuracy ${ }^{(21,22)}$. Fat mass is adjusted to be more accurate: fat mass/(fat mass + lean mass + bone mineral content $) \times 100^{(23)}$. On the day of each test, the equipment was calibrated following the manufacturer's guidelines.

\section{Cardiometabolic measurements}

The study physician drew a blood sample $(8 \mathrm{ml})$ by venepuncture. Plasma or serum was separated by centrifugation within $3 \mathrm{~h}$ after phlebotomy and immediately frozen until analysis to obtain biochemical variables. Colorimetric methods (Cobas c111 analyser; Roche Diagnostics GmbH, Indianapolis, IN, USA) were used for total cholesterol (TC), LDL-cholesterol (LDL-C), HDL-cholesterol (HDL-C), TAG, fasting plasma glucose (FPG) and serum homocysteine 
Table 1 Descriptive characteristics by sex in ninety-three healthy, prepubertal, Ladino children (aged 7-12 years), Guatemala

\begin{tabular}{|c|c|c|c|c|c|c|}
\hline \multirow[b]{2}{*}{ Variable } & \multicolumn{2}{|c|}{ Total $(n 93)$} & \multicolumn{2}{|c|}{ Boys ( $n$ 46) } & \multicolumn{2}{|c|}{ Girls ( $n$ 47) } \\
\hline & Mean or \% & SD or $n$ & Mean or $\%$ & SD or $n$ & Mean or $\%$ & SD or $n$ \\
\hline \multicolumn{7}{|l|}{ Anthropometry } \\
\hline Age (years) & 9.7 & 1.4 & 9.8 & 1.4 & 9.7 & 1.4 \\
\hline Height $(\mathrm{cm})$ & 131.5 & $9 \cdot 8$ & $131 \cdot 0$ & $9 \cdot 8$ & 131.9 & 9.9 \\
\hline Weight $(\mathrm{kg})$ & 33.2 & 9.9 & 33.3 & $10 \cdot 4$ & 33.2 & $9 \cdot 6$ \\
\hline $\operatorname{BMI}\left(\mathrm{kg} / \mathrm{m}^{2}\right)$ & 18.9 & 3.7 & $19 \cdot 0$ & 3.7 & 18.7 & 3.7 \\
\hline BMI-for-age Z-score* & 0.8 & 1.3 & 0.9 & 1.4 & 0.7 & 1.3 \\
\hline Obese, WHO $(\%, n) \dagger$ & 20 & 19 & 20 & 9 & 21 & 10 \\
\hline Obese, CDC $(\%, n) \dagger$ & 18 & 17 & 17 & 8 & 19 & 9 \\
\hline Obese, IOTF $(\%, n) \dagger$ & 13 & 12 & 13 & 6 & 13 & 6 \\
\hline \multicolumn{7}{|l|}{ Body composition (DXA) } \\
\hline \%BF士 & 31.2 & $6 \cdot 6$ & 29.5 & $7 \cdot 0$ & 32.9 & 5.7 \\
\hline Fat mass $(\mathrm{kg})$ & $10 \cdot 7$ & $5 \cdot 1$ & $10 \cdot 2$ & $6 \cdot 4$ & 11.2 & 4.8 \\
\hline \%TF‡ & 29.5 & 9.0 & $27 \cdot 4$ & 9.6 & 31.4 & 8.0 \\
\hline \multicolumn{7}{|l|}{ Metabolic parameters } \\
\hline $\mathrm{TC}(\mathrm{mg} / \mathrm{dl})$ & $149 \cdot 1$ & $32 \cdot 3$ & $147 \cdot 2$ & 34.8 & $151 \cdot 0$ & $30 \cdot 0$ \\
\hline HDL-C (mg/dl) & 41.0 & 11.0 & 42.0 & $10 \cdot 3$ & 40.0 & 11.6 \\
\hline TC:HDL-C & 3.9 & 1.3 & 3.7 & 1.3 & 4.0 & 1.3 \\
\hline TAG $(\mathrm{mg} / \mathrm{dl})$ & $122 \cdot 2$ & 71.8 & 115.5 & 78.9 & 128.8 & 64.3 \\
\hline LDL-C (mg/dl) & 83.7 & $26 \cdot 9$ & $82 \cdot 1$ & $26 \cdot 3$ & 85.2 & $27 \cdot 7$ \\
\hline FPG $(\mathrm{mg} / \mathrm{dl}) \ddagger$ & $81 \cdot 0$ & 6.3 & $82 \cdot 4$ & $5 \cdot 3$ & 79.7 & 7.0 \\
\hline HOMA-IR & 1.4 & 1.0 & 1.3 & 0.9 & 1.5 & $1 \cdot 1$ \\
\hline Serum homocysteine $(\mu \mathrm{mol} / \mathrm{l})$ & 7.5 & $2 \cdot 1$ & 7.7 & $2 \cdot 2$ & 7.4 & $2 \cdot 1$ \\
\hline SBP $(\mathrm{mmHg})$ & 92.7 & $10 \cdot 0$ & 93.6 & 9.6 & 91.8 & 10.4 \\
\hline $\mathrm{DBP}(\mathrm{mmHg})$ & 53.7 & $7 \cdot 0$ & $54 \cdot 1$ & $7 \cdot 3$ & 53.4 & $6 \cdot 8$ \\
\hline
\end{tabular}

CDC, Centers for Disease Control and Prevention; IOTF, International Obesity Task Force; DXA, dual-energy X-ray absorptimetry; \%BF, percentage body fat; \%TF, percentage trunk fat; TC, total cholesterol; HDL-C, HDL-cholesterol; LDL-C, LDL-cholesterol; FPG, fasting plasma glucose; HOMA-IR, homeostasis model assessment-insulin resistance index; SBP, systolic blood pressure; DBP, diastolic blood pressure.

${ }^{*}$ According to the WHO reference ${ }^{(20)}$

†Values reported are percentage $(\%)$ and number of subjects $(n)$ for total, boys and girls.

$\ddagger$ Significantly different by sex $(P<0.05)$.

analyses. Serum insulin was obtained by chemiluminescent enzyme immunoassay (Immulyte 2000; Siemens Healthcare Global, Deerfield, IL, USA). Insulin resistance (homeostasis model assessment-insulin resistance index) was estimated as: $\mathrm{HOMA}-\mathrm{IR}=($ glucose $\times$ insulin$) / 405$ (for glucose in $\mathrm{mg} /$ $\mathrm{dl}$ and insulin in $\mu \mathrm{UI} / \mathrm{ml})^{(24)}$. The derived TC:HDL-C ratio was determined. Blood pressure (BP) was measured in triplicate, at least $1 \mathrm{~min}$ apart, using a standardized technique and an appropriate size for the arm cuff. The mean of the second and third measurements was reported. If the difference between them was $10 \mathrm{mmHg}$ for systolic or diastolic BP, a fourth measurement was obtained, and the mean of the two closest was used.

\section{Definition of adverse cardiometabolic profile}

We considered adverse lipid levels as those referred to in the American Academy of Pediatrics' 2008 report $^{(25)}$, corresponding to 95 th percentile values (5th for HDL-C) for children $<10$ and $\geq 10$ years old. We used the cut-off of $\geq 100 \mathrm{mg} / \mathrm{dl}$ for FPG from the American Diabetes Association $^{(26)}$. HOMA-IR higher than 3.2 was considered abnormal $^{(24)}$. Based on adult observations, TC:HDL-C greater than 4.5 was considered adverse ${ }^{(27,28)}$.

From 6 to 9 years old and from 10 to 12 years old, hypertension was considered as systolic and diastolic BP levels $\geq 122 / 78 \mathrm{mmHg}$ and $\geq 126 / 82 \mathrm{mmHg}$, respectively ${ }^{(29)}$.
The term 'adverse cardiometabolic profile' (ACMP) has been thoroughly studied in relation to the $\mathrm{MetS}^{(30)}$. A definition of MetS is established for adults ${ }^{(31)}$, but remains controversial for children ${ }^{(32)}$. Therefore, an ad hoc definition of risk profile was used. Considering the definitions above, we defined an ACMP from those parameters that proved to have a moderate to good significant correlation with \%BF ( $r=0.50$ to 0.75$)$ in both sexes, which resulted as HOMA-IR higher than 3.2 and/or TC:HDL-C greater than 4.5 (Table 2).

\section{Statistical analyses}

The sex-stratified profile of the sample was described quantitatively. Significant differences across anthropometric and metabolic parameters between sexes were investigated using Student's $t$ test or the Mann-Whitney $U$ test.

The prevalence of adverse lipid profile, TC:HDL-C, FPG, HOMA-IR and BP was estimated. Afterwards, the prevalence of obesity with the WHO (BMI-for-age $Z$-score $>2$ ), International Obesity Task Force (BMI-for-age equivalent to $\geq 30 \mathrm{~kg} / \mathrm{m}^{2}$ in adults) and Centers for Disease Control and Prevention standards (BMI-for-age $\geq 95$ th percentile) was determined $^{(20,33,34)}$. The differences between the three criteria were tested using the $\chi^{2}$ test.

Age- and sex-adjusted Spearman analyses were used to obtain cross-correlations between adiposity (\%BF) and 
Table 2 Cross-correlation between adiposity and BMI with cardiometabolic factors: partial Spearman correlation coefficients adjusted for age and/or sex ${ }^{*}$ in ninety-three healthy, prepubertal, Ladino children (aged 7-12 years), Guatemala

\begin{tabular}{|c|c|c|c|c|c|c|}
\hline \multirow[b]{3}{*}{ Cardiometabolic risk factor } & \multicolumn{3}{|c|}{$\% B F(D X A)$} & \multicolumn{3}{|c|}{ BMI-for-age Z-score } \\
\hline & Total & Boys & Girls & Total & Girls & Boys \\
\hline & $\rho_{\mathrm{p}}{ }^{*}$ & $\rho_{\mathrm{p}}{ }^{*}$ & $\rho_{\mathrm{p}}{ }^{*}$ & $\rho_{\mathrm{p}}{ }^{*}$ & $\rho_{\mathrm{p}}{ }^{*}$ & $\rho_{\mathrm{p}}^{*}$ \\
\hline $\begin{array}{l}\text { TC }(\mathrm{mg} / \mathrm{dl}) \\
\text { HDL-C }(\mathrm{mg} / \mathrm{dl}) \\
\text { TC:HDL-C } \\
\text { TAG (mg/dl) } \\
\text { LDL-C (mg/dl) } \\
\text { FPG (mg/dl) } \\
\text { Serum insulin }(\mu \mathrm{Ul} / \mathrm{ml}) \\
\text { HOMA-IR } \\
\text { Serum homocysteine }(\mu \mathrm{mol} / \mathrm{l}) \\
\text { SBP }(\mathrm{mmHg}) \\
\text { DBP }(\mathrm{mmHg})\end{array}$ & $\begin{array}{l}0.33 \\
-0.37 \\
0.60 \\
0.55 \\
0.26 \\
0.08 \dagger \\
0.64 \\
0.64 \\
0.07 \dagger \\
0.37 \\
0.47\end{array}$ & $\begin{array}{c}0.39 \\
-0.28 \dagger \\
0.58 \\
0.49 \\
0.33 \\
0.07 \dagger \\
0.59 \\
0.60 \\
0.04 \dagger \\
0.43 \\
0.57\end{array}$ & $\begin{array}{l}0.25 \dagger \\
-0.45 \\
0.62 \\
0.62 \\
0.17 \dagger \\
0.23 \dagger \\
0.70 \\
0.69 \\
0.16 \dagger \\
0.41 \\
0.41\end{array}$ & $\begin{array}{l}0.20 \dagger \\
-0.40 \\
0.55 \\
0.44 \\
0.15 \dagger \\
0.20 \dagger \\
0.61 \\
0.61 \\
0.05 \dagger \\
0.54 \\
0.51\end{array}$ & $\begin{array}{c}0.30 \\
-0.30 \dagger \\
0.56 \\
0.36 \\
0.24 \dagger \\
0.12 \dagger \\
0.62 \\
0.61 \\
-0.01 \dagger \\
0.52 \\
0.57\end{array}$ & $\begin{array}{l}0.08 \dagger \\
-0.52 \\
0.55 \\
0.56 \\
0.07 \dagger \\
0.24 \dagger \\
0.68 \\
0.66 \\
0.09 \dagger \\
0.48 \\
0.43\end{array}$ \\
\hline
\end{tabular}

\%BF, percentage body fat; DXA, dual-energy X-ray absorptimetry; TC, total cholesterol; HDL-C, HDL-cholesterol; LDL-C, LDL-cholesterol; FPG, fasting plasma glucose; HOMA-IR, homeostasis model assessment-insulin resistance index; SBP, systolic blood pressure; DBP, diastolic blood pressure.

${ }^{*} \rho_{\mathrm{p}}$, Spearman correlation coefficients adjusted for age and/or sex.

†No significant correlation $(P \geq 0.05)$.

BMI-for-age $Z$-scores with cardiometabolic risk parameters (lipid profile, insulin resistance and $\mathrm{BP}$ ).

A threshold of \%BF that predicted an ACMP with a receiver operating characteristic (ROC) curve was determined ${ }^{(35)}$. The sample distribution of \%BF was divided into $2 \%$ thresholds. For each threshold, we calculated sensitivity (percentage of all children with an ACMP defined as obese by the threshold), specificity (percentage of all children without an ACMP defined as non-obese by the threshold), positive likelihood ratio (LHR; sensitivity/(1-specificity), the odds of having an ACMP above the threshold) and negative LHR ((1-sensitivity)/specificity, the odds of not having an ACMP below the threshold $)^{(36)}$. According to the ROC curve analysis, a threshold based on the maximization of sensitivity and specificity was selected. The area under the curve (AUC) was calculated with the parametric ROC curve analysis method, suggesting the accuracy of \%BF to discriminate between children with and without the risk profile.

The explained ROC curve analyses for BMI-for-age $Z$-scores and $\% \mathrm{BF}$ were repeated to obtain the surrogate threshold of BMI $Z$-score that divided our sample into children above and below the previously defined \%BF threshold. In this case, BMI-for-age $Z$-scores were divided into thresholds of $0 \cdot 5$.

Statistical analyses were performed using the statistical software package STATA SE version $12 \cdot 1$. Statistical significance was defined as $P<0 \cdot 05$.

\section{Results}

Mean age of the participants was 9.7 (SD 1.4) years. The prevalence of elevated TC, TC:HDL-C, TAG, LDL-C, FPG and HOMA-IR levels was $7 \cdot 5 \%, 10 \cdot 8 \%, 54.8 \%, 3.2 \%$, $1 \cdot 1 \%$ and $5.4 \%$, respectively. No child was hypertensive,
$43 \%$ had low HDL-C and $43 \%$ were overweight (BMI-forage $Z$-score $>1$ ). The prevalence of obesity varied from $20 \%$ under the WHO reference to $19 \%$ by the Centers for Disease Control and Prevention and $13 \%$ by the International Obesity Task Force standards $(P<0 \cdot 001) . \% \mathrm{BF}$ and \%TF were both significantly higher in girls (Table 1 ).

HOMA-IR and TC:HDL-C had the highest association with \%BF ( $r=0.64$ and 0.60 , respectively; Table 2$)$. Correlations of these two and most cardiometabolic risk factors were somewhat lower with BMI-for-age $Z$-scores than with $\% \mathrm{BF}$. Correlation of BMI-for-age $Z$-score and $\% \mathrm{BF}$ was high $(r=0 \cdot 84, P<0 \cdot 001)$. The prevalence of the ACMP profile was $16 \%$.

Table 3 shows the specificity, sensitivity, positive and negative LHR for each \%BF threshold in the prediction of an ACMP. The \%BF threshold that maximized sensitivity and specificity was $36 \%$. The prevalence of children having $\mathrm{BF} \geq 36 \%$ was $27 \%$, who were five times more likely to have an ACMP compared with those having BF $<36 \%$. The AUC for the \%BF data was 0.93 (sD 0.04; Fig. 1), indicating excellent accuracy of \%BF predicting an ACMP.

Table 4 shows the specificity, sensitivity, positive and negative LHR for each BMI-for-age $Z$-score threshold in the prediction of excess adiposity, defined by the selected threshold of $\mathrm{BF} \geq 36 \%$. A BMI-for-age $Z$-score threshold of 1.5 was selected as it maximized sensitivity and specificity. The prevalence of children having BMI-for-age $Z$-score $\geq 1.5$ was $32 \%$, who were eight times more likely to have excess adiposity compared with those having BMI-for-age $Z$-score $<1.5$. Children with a BMI-for-age $Z$-score $<1.5$ were $86 \%$ less likely to have excess adiposity. The AUC for the BMI data was 0.95 (SD 0.02; Fig. 2), indicating excellent accuracy of BMI-for-age $Z$-score in predicting the critical $\%$ BF that implies an ACMP. 
Table 3 Specificity, sensitivity and likelihood ratios for each \%BF threshold to predict an adverse cardiometabolic risk profile in ninety-three healthy, prepubertal, Ladino children (aged 7-12 years), Guatemala

\begin{tabular}{lcccc}
\hline$\% B F$ range & Sensitivity & Specificity & LHR $_{\text {pos }} \dagger$ & LHR $_{\text {neg }} \ddagger$ \\
\hline$<18.0$ & 1.00 & 0.00 & 1.00 & - \\
$18.0-19.9$ & 1.00 & 0.01 & 1.01 & 0.00 \\
$20.0-21.9$ & 1.00 & 0.04 & 1.04 & 0.00 \\
$22.0-23.9$ & 1.00 & 0.07 & 1.08 & 0.00 \\
$24.0-25.9$ & 1.00 & 0.14 & 1.16 & 0.00 \\
$26.0-27.9$ & 1.00 & 0.27 & 1.37 & 0.00 \\
$28.0-29.9$ & 1.00 & 0.38 & 1.62 & 0.00 \\
$30.0-31.9$ & 1.00 & 0.53 & 2.13 & 0.00 \\
$32.0-33.9$ & 0.92 & 0.63 & 2.48 & 0.13 \\
$34.0-35.9$ & 0.92 & 0.79 & 4.37 & 0.11 \\
$36.0-37.9$ & 0.92 & 0.83 & 5.30 & 0.10 \\
$38.0-39.9$ & 0.75 & 0.90 & 7.59 & 0.28 \\
$40.0-41.9$ & 0.67 & 0.96 & 18.00 & 0.35 \\
$42.0-43.9$ & 0.33 & 0.98 & 13.50 & 0.68 \\
$44.0-45.9$ & 0.17 & 1.00 & $\infty$ & 0.83 \\
$\geq 46.0$ & 0.00 & 1.00 & $\infty$ & 1.00 \\
\hline
\end{tabular}

$\% \mathrm{BF}$, body fat percentage; LHR, likelihood ratio; pos, positive; neg, negative; HOMA-IR, homeostasis model assessment-insulin resistance index TC, total cholesterol; HDL-C, HDL-cholesterol.

${ }^{*}$ For each \%BF demarcation range, adverse CVD risk profile is defined as HOMA-IR $\geq 3.16$ and/or TC:HDL-C $\geq 4.5$

†Sensitivity/( 1 - specificity).

$\ddagger(1-$ Sensitivity $) /$ specificity.

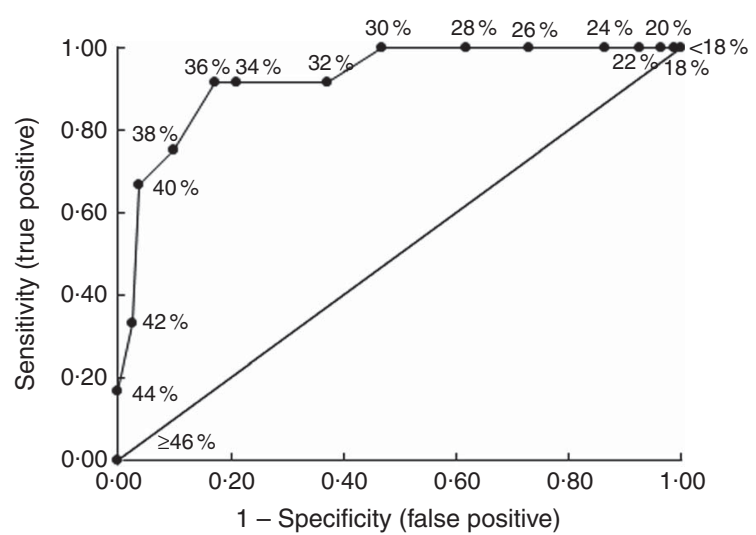

Fig. 1 Empirical receiver operating characteristic curve predicting children with abnormal homeostasis model assessment-insulin resistance index and/or total cholesterol: HDL-cholesterol levels using specific percentage body fat thresholds in ninety-three healthy, prepubertal, Ladino children (aged $7-12$ years), Guatemala

\section{Discussion}

The BMI $Z$-score cut-off point of 1.5 was estimated from a $36 \%$ BF threshold built from adverse cardiometabolic parameters with which the correlation was moderate to good. That resulted in the best trade-off between sensitivity and specificity for Guatemalan Ladino children to predict excess adiposity compromising health. Following the WHO classification, eleven out of ninety-three children would have been misclassified downwards with the BMI $Z$-score cut-off of $>2$ (sensitivity $64 \%$ ); and nine out of ninety-three would have been misclassified upwards with the BMI $Z$-score cut-off of $>1$ (sensitivity $100 \%$ ).
Table 4 Specificity, sensitivity and likelihood ratios for each BMI-for-age Z-score threshold to predict adiposity in ninety-three healthy, prepubertal, Ladino children (aged $7-12$ years), Guatemala

\begin{tabular}{lcccc}
\hline $\begin{array}{l}\text { BMl-for-age } \\
\text { Z-score range* }\end{array}$ & Sensitivity & Specificity & LHR $_{\text {pos }} \dagger$ & LHR $_{\text {neg }} \ddagger$ \\
\hline$<-1.00$ & 1.00 & 0.00 & 1.00 & - \\
-1.00 to -0.51 & 1.00 & 0.06 & 1.06 & 0.00 \\
-0.50 to -0.01 & 1.00 & 0.26 & 1.36 & 0.00 \\
0.00 to 0.49 & 1.00 & 0.46 & 1.84 & 0.00 \\
0.50 to 0.99 & 1.00 & 0.65 & 2.83 & 0.00 \\
1.00 to 1.49 & 1.00 & 0.78 & 4.53 & 0.00 \\
1.50 to 1.99 & 0.88 & 0.88 & 7.48 & 0.14 \\
2.00 to 2.49 & 0.64 & 0.96 & 14.51 & 0.38 \\
2.50 to 2.99 & 0.40 & 0.97 & 13.60 & 0.62 \\
3.00 to 3.49 & 0.24 & 1.00 & $\infty$ & 0.76 \\
3.50 to 3.99 & 0.08 & 1.00 & $\infty$ & 0.92 \\
$\geq 4.00$ & 0.00 & 1.00 & $\infty$ & 1.00 \\
\hline
\end{tabular}

LHR, likelihood ratio; pos, positive; neg, negative; BF, body fat. *BMI-for-age $Z$-score demarcation ranges according to the WHO reference ${ }^{(20)}$. For each range, $36 \% \mathrm{BF}$ is the cut-off point defining excess of adiposity implying risk profile, as revealed in Table 3.

†Sensitivity/( 1 - specificity).

$\ddagger(1-$ Sensitivity $) /$ specificity.

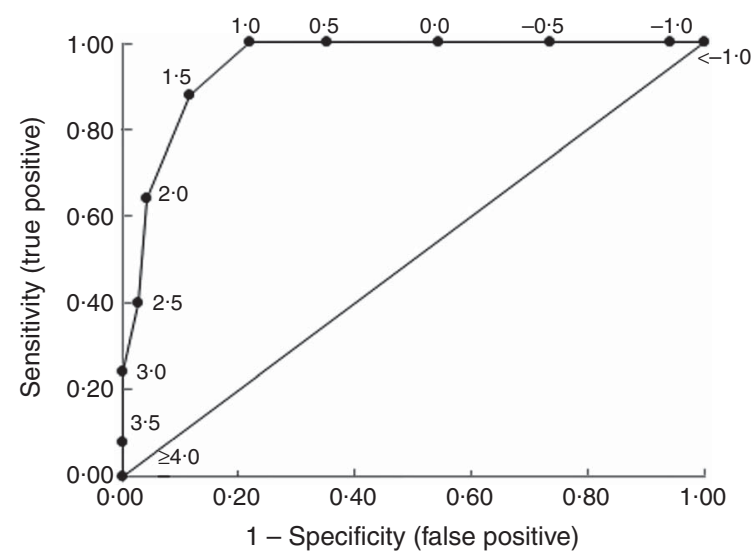

Fig. 2 Empirical receiver operating characteristic curve predicting children with excess adiposity levels implying an adverse cardiometabolic profile using specific BMI-for-age Z-score thresholds in ninety-three healthy, prepubertal, Ladino children (aged 7-12 years), Guatemala

Most studies have evaluated excess adiposity according to a sex- and age-specific $\%$ BF percentile ${ }^{(16,37,38)}$. Three heterogeneous studies proposed \%BF cut-offs in childhood based on cross-sectional associations with CVD risk factors $^{(14,15,39)}$. They differed in the selection of the risk factors' threshold levels.

The MetS is a cluster of risk factors for CVD and type 2 diabetes $^{(40)}$. Obesity plays a primary role and leads to insulin resistance, increased $\mathrm{BP}$ and dyslipidaemia ${ }^{(31)}$. Its definition for children is problematic because of changes in lipid profile, BP, insulin sensitivity and anthropometry with age and pubertal development. Thus, several cut-off values for the risk factors have been used ${ }^{(41)}$. The International Diabetes Federation made a proposal, but also suggested that MetS should not be diagnosed as an entity 
in children younger than 10 years $^{(42)}$. Therefore, we defined an ad hoc ACMP based on the clustering of risk factors and their correlation with \%BF. Insulin resistance is a major precursor of CVD and type 2 diabetes $^{(30)}$ and among children, the reliability of HOMA-IR for its measurement is well established ${ }^{(24)}$. Moreover, the ratio TC:HDL-C is a positive predictor of oxidized LDL concentrations, playing an important role in the atherosclerotic process ${ }^{(28)}$.

Comparison of studies that explore the feasibility of overweight to identify fat children is challenging, as different BMI and adiposity cut-offs are proposed ${ }^{(17,43,44)}$. Moreover, a high BMI level is a specific, but not a sensitive, predictor of $\% \mathrm{BF}^{(37,43,45)}$. Among children, BMI has inconsistently been correlated with adiposity ${ }^{(2,46)}$ and its accuracy increases with the level of $\mathrm{BF}^{(2,47,48)}$. Then, the high correlation found between $\mathrm{BMI}$ and \%BF in the present study might be due to our oversampling of overweight children. However, some ethnic groups, across all levels of BMI, have higher $\% \mathrm{BF}^{(9,10)}$. Our $36 \%$ $\mathrm{BF}$ threshold, higher than those of Higgins, Williams or Dwyer $^{(14,15,39)}$, suggests that Guatemalan Ladino children may have higher \%BF basal levels. Although there is a significant association between overweight classification by WHO and cardiometabolic risk ${ }^{(49)}$, BMI misclassifies individuals with increased risk related to elevated $\operatorname{adiposity}^{(50)}$.

BMI cut-offs detecting excess adiposity and \%BF thresholds detecting risk profile with excellent discrimination were assessed $^{(51)}$. In the whole spectrum of possible decision thresholds, we selected those optimizing both sensitivity and specificity. The positive LHR for the BMI-for-age $Z$-score of $2 \cdot 0$ doubles that of the 1.5 cut-off, and would therefore be more accurate in showing the likelihood of having ACMP in those with elevated adiposity, but at the cost of worsening the sensitivity (Table 4). However, the health care of children puts a greater emphasis on more sensitive references for prevention tasks. Instead, the WHO BMI $Z$-score of $>1$ would increase the false-positive rate.

We acknowledge several important limitations of the present study. About half of the children were preselected to be overweight, so we cannot know the real prevalence of overweight/obesity. Moreover, Tanner stage was not assessed, so we could expect an influence of sex on biochemistry and \%BF. To minimize this, we chose BMIfor-age $Z$-scores, which are sex- and age-adjusted, and lipid profile cut-offs above and below the age of 10 years, because most children below 10 years old are in Tanner stage 1 (prepubertal) ${ }^{(29)}$. The proposed risk profile definition is a modest approach to the multifaceted obese phenotype, lacking the dynamic view of muscle and fat $^{(40)}$. Nevertheless, empirical categories are required for clinical practice. Estimating cut-off points for boys and girls separately would be the preferred choice but our limited sample size precluded it. Finally, parametric ROC curve analyses have the drawback of discarding data when they are grouped $^{(35)}$. Therefore, ROC curve points and the AUC may be biased. However, as ROC curve analysis is independent of prevalence ${ }^{(35)}$, we had the advantage of equal numbers of participants with both conditions to evaluate without the need for representative samples.

Another limitation comes from the cross-sectional design of the study and the inherent lack of temporality in our causal hypothesis, but several colleagues ${ }^{(14,15,39)}$ have previously used cross-sectional data to develop \%BF cut-off points for defining obesity implying cardiometabolic risk.

Internal validity may somehow be affected in our study by the fact that the BMI $Z$-score threshold of 1.5 is based on a $36 \%$ BF threshold, implying a false-negative rate of $8 \%$ (92\% sensitivity) in the first step, and in our final step we found a false-negative rate of $12 \%$ ( $88 \%$ sensitivity). Like Higgins et al. ${ }^{(14)}$, we used dual-energy X-ray absorptiometry to obtain a robust estimation of $\% \mathrm{BF}$, as this technique is not affected by intra- and inter-observer variability.

According to our criteria of ACMP, ten children (10.8\% of the sample) had TC:HDL-C $>4.5$ and five children (5.4\%) presented a HOMA-IR $>3 \cdot 2$; altogether, fifteen children. It is difficult to quantify the validity and reliability of these thresholds because there is no clear standard definition of $\mathrm{ACMP}^{(41)}$.

Nevertheless, to our knowledge, this is the first attempt to assess the accuracy of BMI as an indicator of the degree of harmful adiposity using a two-step method. It represents an approach to obesity classification and a novel contribution to the literature concerning MetS.

\section{Conclusion}

In summary, as current BMI definitions have limitations in screening for adiposity, further efforts to build specific BMI cut-off points derived from ethnic-/sex- and age-specific $\% \mathrm{BF}$ thresholds remain necessary. Such tailored tools may help developing programmes aimed to identify early metabolic risk in other child populations.

\section{Acknowledgements}

Acknowledgements: The study was accomplished thanks to four collaborating institutions: the School of Medicine at the University of Alcalá in Spain; INCAP in Guatemala; the Department of Epidemiology at Michigan University; and the Department of International Health at the Johns Hopkins Bloomberg School of Public Health. The authors thank all the study participants, parents and children in the two schools in Guatemala. They also thank all the technicians who performed the fieldwork measurements at the Physiology and Body Composition Laboratory of CIIPEC. Financial support: This project was funded in part with 
Federal funds from the National Heart, Lung, and Blood Institute (NHLBI), National Institutes of Health, Department of Health and Human Services (under contract no. HHSN26820900032C). The NHLBI had no role in the design, analysis or writing of this article. Conflicts of interest: None. Authorship: O.R., M.F. and E.V. contributed to the original design. M.R.-Z., D.R. and F.K. organized and conducted data collection. O.R. reorganized, reordered and recoded variables of the database. O.R., E.V., U.B. and M.F. conducted the statistical analyses. O.R., E.V., U.B., B.C., M.R.-Z. and M.F. were active in the interpretation of results. The manuscript was drafted by O.R., M.F., U.B. and E.V. and reviewed by all authors. All authors have approved the final report. All authors had full access to the data in the study and take responsibility for its integrity and the accuracy of the data analysis. M.F. is the guarantor and corresponding author for this study. Ethics of human subject participation: This study was approved by the INCAP's Institutional Ethics Committee.

\section{References}

1. International Obesity Taskforce (2012) Obesity \& Research/ Obesity the Global Epidemic. http://www.iaso.org/iotf/ obesity/obesitytheglobalepidemic/ (accessed October 2012).

2. Centers for Disease Control and Prevention (2012) Childhood Overweight and Obesity. http://www.cdc.gov/obesity/ childhood/index.html (accessed October 2012).

3. Mbowe O, Díaz A, Wallace J et al. (2013) Prevalence of metabolic syndrome and associated cardiovascular risk factors in Guatemalan school children. Matern Child Health $J$ (Epublication ahead of print version).

4. Freedman DS, Ogden CL, Berenson GS et al. (2005) Body mass index and body fatness in childhood. Curr Opin Clin Nutr Metab Care 8, 618-623.

5. Lazarus R, Baur L, Webb K et al. (1996) Body mass index in screening for adiposity in children and adolescents: systematic evaluation using receiver operating characteristic curves. Am J Clin Nutr 63, 500-506.

6. Taylor RW, Jones IE, Williams SM et al. (2002) Body fat percentages measured by dual-energy X-ray absorptiometry corresponding to recently recommended body mass index cutoffs for overweight and obesity in children and adolescents aged 3-18 y. Am J Clin Nutr 76, 1416-1421.

7. Veiga GV, Dias PC \& Anjos LA (2001) A comparison of distribution curves of body mass index from Brazil and the United States for assessing overweight and obesity in Brazilian adolescents. Pan Am J Public Health 10, 79-85.

8. Brann LS (2008) Classifying preadolescent boys based on their weight status and percent body fat produces different groups. J Am Diet Assoc 108, 1018-1022.

9. Flegal KM, Ogden CL, Yanovski JA et al. (2010) High adiposity and high body mass index-for-age in US children and adolescents overall and by race-ethnic group. $A m J$ Clin Nutr 91, 1020-1026.

10. Deurenberg-Yap M, Schmidt G, Van Staveren WA et al. (2000) The paradox of low body mass index and high body fat percentage among Chinese, Malays and Indians in Singapore. Int J Obes Relat Metab Disord 24, 1011-1017.

11. Okorodudu DO, Jumean MF, Montori VM et al. (2010) Diagnostic performance of body mass index to identify obesity as defined by body adiposity: a systematic review and meta-analysis. Int J Obes (Lond) 34, 791-799.
12. Kurtoglu S, Mazicioglu MM, Ozturk A et al. (2010) Body fat reference curves for healthy Turkish children and adolescents. Eur J Pediatr 169, 1329-1335.

13. Biro FM \& Wien M (2010) Childhood obesity and adult morbidities. Am J Clin Nutr 91, issue 5, 1499S-1505S.

14. Higgins PB, Gower BA, Hunter GR et al. (2001) Defining health-related obesity in prepubertal children. Obes Res $\mathbf{9}$, 233-240.

15. Williams DP, Going SB, Lohman TG et al. (1992) Body fatness and risk for elevated blood pressure, total cholesterol, and serum lipoprotein ratios in children and adolescents. Am J Public Health 82, 358-363.

16. Li L, Pinot de Moira A \& Power C (2011) Predicting cardiovascular disease risk factors in midadulthood from childhood body mass index: utility of different cutoffs for childhood body mass index. Am J Clin Nutr 93, 1204-1211.

17. Monasta L, Lobstein T, Cole TJ et al. (2011) Defining overweight and obesity in pre-school children: IOTF reference or WHO standard? Obes Rev 12, 295-300.

18. Twells LK \& Newhook LA (2011) Obesity prevalence estimates in a Canadian regional population of preschool children using variant growth references. BMC Pediatr 11, 21.

19. Shields M \& Tremblay MS (2010) Canadian childhood obesity estimates based on WHO, IOTF and CDC cut-points. Int J Pediatr Obes 5, 265-273.

20. World Heath Organization (2012) Growth Reference 5-19 years. BMI-for-age. http://www.who.int/growthref/en/ (accessed October 2012).

21. Hangartner TN, Warner S, Braillon P et al. (2013) The Official Positions of the International Society for Clinical Densitometry: acquisition of dual-energy X-ray absorptiometry body composition and considerations regarding analysis and repeatability of measures. J Clin Densitom 16, 520-536.

22. Fields DA \& Goran MI (2000) Body composition techniques and the four-compartment model in children. J Appl Physiol 89, 613-620.

23. Goran MI, Driscoll P, Johnson R et al. (1996) Crosscalibration of body-composition techniques against dualenergy X-ray absorptiometry in young children. Am J Clin Nutr 63, 299-305.

24. Keskin M, Kurtoglu S, Kendirci M et al. (2005) Homeostasis model assessment is more reliable than the fasting glucose/ insulin ratio and quantitative insulin sensitivity check index for assessing insulin resistance among obese children and adolescents. Pediatrics 115, e500-e503.

25. Daniels SR \& Greer FR (2008) Lipid screening and cardiovascular health in childhood. Pediatrics 122, 198-208.

26. American Diabetes Association (2006) Diagnosis and classification of diabetes mellitus. Diab Care 29, Suppl. 1, S43-S48.

27. Ingelsson E, Schaefer EJ, Contois JH et al. (2007) Clinical utility of different lipid measures for prediction of coronary heart disease in men and women. JAMA 298, 776-785.

28. Barbosa KB, Volp AC, Hermsdorff HH et al. (2011) Relationship of oxidized low density lipoprotein with lipid profile and oxidative stress markers in healthy young adults: a translational study. Lipids Health Dis 10, 61.

29. Horan MJ \& Sinaiko AR (1987) Synopsis of the report of the second task force in blood pressure control in children. Hypertension 10, 115-121.

30. Rodrigues AN, Abreu GR, Resende RS et al. (2013) Cardiovascular risk factor investigation: a pediatric issue. Int J Gen Med 6, 57-66.

31. Alberti KG, Zimmet P \& Shaw J (2006) Metabolic syndrome a new world-wide definition. A Consensus Statement from the International Diabetes Federation. Diabet Med 23, 469-480.

32. Gustafson JK, Yanoff LB, Easter BD et al. (2009) The stability of metabolic syndrome in children and adolescents. J Clin Endocrinol Metab 94, 4828-4834. 
33. Cole TJ, Bellizzi MC, Flegal KM et al. (2000) Establishing a standard definition for child overweight and obesity worldwide: international survey. Br Med J 320, 1240-1243.

34. Centers for Disease Control and Prevention (2012) Children's BMI Group calculator (metric version). http://www.cdc.gov/ healthyweight/assessing/bmi/childrens_bmi/tool_for_schools. html (accessed November 2012).

35. Søreide K (2009) Receiver-operating characteristic curve analysis in diagnostic, prognostic and predictive biomarker research. J Clin Patbol 62, 1-5.

36. Zhou X-H, Obuchowski NA \& McClish DK (2002) Statistical Methods in Diagnostic Medicine. New York: WileyInterscience.

37. Zimmermann MB, Gubeli C, Puntener C et al. (2004) Detection of overweight and obesity in a national sample of 6-12-y-old Swiss children: accuracy and validity of reference values for body mass index from the US Centers for Disease Control and Prevention and the International Obesity Task Force. Am J Clin Nutr 79, 838-843.

38. Mei Z, Grummer-Strawn LM, Pietrobelli A et al. (2002) Validity of body mass index compared with other bodycomposition screening indexes for the assessment of body fatness in children and adolescents. Am J Clin Nutr 75, 978-985.

39. Dwyer T \& Blizzard CL (1996) Defining obesity in children by biological endpoint rather than population distribution. Int J Obes Relat Metab Disord 20, 472-480.

40. Müller MJ, Lagerpusch M, Enderle J et al. (2012) Beyond the body mass index: tracking body composition in the pathogenesis of obesity and the metabolic syndrome. Obes Rev 13, 6-13.

41. Reinehr T, de Sousa G, Toschke AM et al. (2007) Comparison of metabolic syndrome prevalence using eight different definitions: a critical approach. Arch Dis Child 92, 1067-1072.
42. Zimmet P, Alberti K, George MM et al.; IDF Consensus Group (2007) The metabolic syndrome in children and adolescents an IDF Consensus Report. Pediatr Diabetes 8, 299-306.

43. Reilly JJ, Dorosty AR, Emmett PM; ALSPAC Study Team (2000) Identification of the obese child: adequacy of the body mass index for clinical practice and epidemiology. Int J Obes Relat Metab Disord 24, 1623-1627.

44. Neovius M, Rössner SM, Vågstrand K et al. (2009) Adiposity measures as indicators of metabolic risk factors in adolescents. Obes Facts 2, 294-301.

45. Wickramasinghe VP, Cleghorn GJ, Edmiston KA et al. (2005) Validity of BMI as a measure of obesity in Australian white Caucasian and Australian Sri Lankan children. Ann Hum Biol 32, 60-71.

46. Wells JC (2001) A critique of the expression of paediatric body composition data. Arch Dis Child 85, 67-72.

47. Widhalm K, Schonegger K, Huemer C et al. (2001) Does the BMI reflect body fat in obese children and adolescents? A study using the TOBEC method. Int J Obes Relat Metab Disord 25, 279-285.

48. Bray GA, DeLany JP, Harsha DW et al. (2001) Evaluation of body fat in fatter and leaner 10-y-old African American and white children: the Baton Rouge Children's Study. Am J Clin Nutr 73, 687-702.

49. de Onis M, Martínez-Costa C, Núñez F et al. (2013) Association between WHO cut-offs for childhood overweight and obesity and cardiometabolic risk. Public Health Nutr 16, 625-630.

50. Gómez-Ambrosi J, Silva C, Galofré JC et al. (2012) Body mass index classification misses subjects with increased cardiometabolic risk factors related to elevated adiposity. Int J Obes (Lond) 36, 286-294.

51. Simundic AM (2008) Measures of diagnostic accuracy: basic definitions. Med Biol Sci 22, 61-65. 